\title{
Self-averaging characteristics of spectral fluctuations
}

\author{
Petr Braun ${ }^{1,2}$ and Fritz Haake田 \\ ${ }^{1}$ Fakultät für Physik, Universität Duisburg-Essen, 47048 Duisburg, Germany and \\ ${ }^{2}$ Institute of Physics, Saint-Petersburg University, 198504 Saint-Petersburg, Russia
}

\begin{abstract}
The spectral form factor as well as the two-point correlator of the density of (quasi-)energy levels of individual quantum dynamics are not self-averaging. Only suitable smoothing turns them into useful characteristics of spectra. We present numerical data for a fully chaotic kicked top, employing two types of smoothing: one involves primitives of the spectral correlator, the second a small imaginary part of the quasi-energy. Self-averaging universal (like the CUE average) behavior is found for the smoothed correlator, apart from noise which shrinks like $\frac{1}{\sqrt{N}}$ as the dimension $N$ of the quantum Hilbert space grows. There are periodically repeated quasi-energy windows of correlation decay and revival wherein the smoothed correlation remains finite as $N \rightarrow \infty$ such that the noise is negligible. In between those windows (where the CUE averaged correlator takes on values of the order $\frac{1}{N^{2}}$ ) the noise becomes dominant and self-averaging is lost. We conclude that the noise forbids distinction of CUE and GUE type behavior. Surprisingly, the underlying smoothed generating function does not enjoy any self-averaging outside the range of its variables relevant for determining the two-point correlator (and certain higher-order ones). - We corroborate our numerical findings for the noise by analytically determining the CUE variance of the smoothed single-matrix correlator.
\end{abstract}

PACS numbers: $05.45 . \mathrm{Mt}$, 03.65.Sq

\section{INTRODUCTION}

Recent semiclassical work based on Gutzwiller's periodic-orbit theory has revealed universal spectral fluctuations for quantum dynamics with a fully chaotic classical limit [1] 6 . However, the present semiclassical theory leaves not satisfactorily answered the question whether universal behavior prevails only under the protection of suitable averages over ensembles of quantum systems which all share the same classical limit (" $\hbar$-averages").

We have therefore thought desirable a thorough investigation of spectral fluctuations of individual quantum dynamics and have chosen a kicked top without time reversal invariance for a case study. As is well known, the spectral form factor $K(n)=\frac{1}{N}\left|\operatorname{Tr} U^{n}\right|^{2}$ (where $n=1,2, \ldots$ is a dimensionless discrete time and $N$ the dimension of the Floquet matrix $U$ ) as well as the two-point correlator $C(e)$ of the density of levels (the Fourier transform of $K(n)$ ) need smoothing in order to become self-averaging indicators of universal spectral fluctuations (or absence thereof) [7 9]. We have checked a certain second primitive of the form factor to be self-averaging and faithful to the average over the circular unitary ensemble (CUE) for times $n$ not negligibly small compared to the Heisenberg time $N$. The correlator, a periodic function of a quasi-energy variable $e$ conjugate to the time $n$, has self-averaging first and second primitives inside certain quasi-energy windows within which correlations have not yet subsided to "noise". Outside those windows of correlation decay and revival (which are tiny in width compared to the period of $C(e)$ ), the noise (of order $N^{-1 / 2}$ ) overwhelms the CUE average (order $\frac{1}{N^{2}}$ for the correlator, $\frac{1}{N}$ for its first primitive) and self-averaging ceases to reign. We are led to the same conclusion when smoothing the correlator by allowing for an imaginary part Ime $>0$, the latter sufficiently large (see below) but smaller than the mean spacing of the eigenphases of $U$.

We represent the correlator as a descendant of a generating function $\mathcal{Z}\left(e, \delta_{+}, \delta_{-}\right)$, a periodic function of three variables. Only the behavior near $\delta_{ \pm}=0$ determines the correlator $C(e)$. A primitive of $\mathcal{Z}$ w.r.t. $e$ turns out smooth, self-averaging, and faithful to the CUE average for an individual kicked top, for $\delta_{ \pm}$near zero and as long as $e$ remains in the windows of correlation decay and revival. Outside, however, there is no self-averaging. Inasmuch as such absence of universality is irrelevant for correlator and form factor (and certain higher-order kins, see below) one might dispatch it as physically uninteresting. Nonetheless, the question arises as to why previous semiclassical work has yielded the RMT generating function without manifest necessity for ensemble averaging. We confirm our numerical findings for the role of noise in Sect. VI by analytically determinating the CUE variance of the smoothed single- $U$ correlator, building on results of Conrey et al for higher-order generating functions [10. We thus generalize the previously known 'ergodicity' of the correlator within the CUE.

*Electronic address: petr.braun@uni-due.de 
The Floquet operator $U=\exp \left(-\mathrm{i} \frac{\tau_{z} J_{z}^{2}}{2 j+1}-\mathrm{i} \alpha_{z} J_{z}\right) \exp \left(-\mathrm{i} \frac{\tau_{y} J_{y}^{2}}{2 j+1}-\mathrm{i} \alpha_{y} J_{y}\right) \exp \left(-\mathrm{i} \frac{\tau_{x} J_{x}^{2}}{2 j+1}-\mathrm{i} \alpha_{x} J_{x}\right)$ of our kicked top lacks time reversal invariance. The rotation angles were chosen as $\alpha_{y}=\alpha_{y}=1, \alpha_{x}=1.1$ and the torsion strengths as $\tau_{z}=10, \tau_{y}=0, \tau_{x}=4$, to bring about predominance of classical chaos; islands of regular motion cumulatively cover less than a single Planck cell. The angular momenta $J_{x / y / z}$ obey $\left[J_{x}, J_{y}\right]=\mathrm{i} J_{z}$ etc.. The quantum number $j$ fixes the dimension of the quantum Hilbert space as $N=2 j+1$; our calculations involve values of $j$ between $10^{2}$ and $10^{4}$.

\section{GENERATING FUNCTION, GENERALIZED CORRELATOR AND FORM FACTOR}

The analysis of spectral fluctuations of unitary quantum maps is conveniently based on the generating function

$$
\mathcal{Z}(a, b, c, d)=\frac{1}{2 \pi} \int_{0}^{2 \pi} \frac{\operatorname{det}\left(1-c \mathrm{e}^{\mathrm{i} \phi} U\right) \operatorname{det}\left(1-d \mathrm{e}^{-\mathrm{i} \phi} U^{\dagger}\right)}{\operatorname{det}\left(1-a \mathrm{e}^{\mathrm{i} \phi} U\right) \operatorname{det}\left(1-b \mathrm{e}^{-\mathrm{i} \phi} U^{\dagger}\right)} d \phi, \quad|a|,|b|<1, \quad c, d \in \mathbb{C} .
$$

That function [6, 11] generates the two-point correlator of the eigenphase density of $U$ as $C(e)=$ $\left.\frac{2 c d}{N^{2}} \partial_{c} \partial_{d} \mathcal{Z}\right|_{a=b=c=d=\exp \left(\mathrm{i} \frac{e}{N}\right)}$.

The center-phase average entails interesting properties. If we set $a=c$, two of the spectral determinants in 1 ) cancel. The $\phi$-integral then simply yields $\mathcal{Z}(a, b, a, d)=1$, as is easily checked by going to a complex $\phi$-plane and complementing the integral to one over a suitable closed loop not encircling the poles provided by the eigenphases of $U$. Similarly, $\mathcal{Z}(a, b, c, b)=1$ such that we have $\mathcal{Z}-1=(a-c)(b-d)[\ldots]$ with [...] a polynomial of order $N-1$ in both $c$ and $d$. In fact, the quantity $\frac{\mathcal{Z}-1}{(a-c)(b-d)}=[\ldots]$ yields the complex two-point correlator as

$$
C(e)=\left.\frac{2 a b}{N^{2}} \frac{\mathcal{Z}-1}{(a-c)(b-d)}\right|_{a=b=c=d=\mathrm{e}^{\mathrm{i} e / N}} .
$$

The foregoing result suggests to define an algebraic kinsman of the generating function to be called generalized correlator,

$$
\mathcal{C}=\left(\frac{a b}{c d}\right)^{\frac{N-1}{2}} \frac{2 a b}{N^{2}} \frac{\mathcal{Z}-1}{(a-c)(b-d)}
$$

which yields the correlator as $C(e)=\left.\mathcal{C}\right|_{a=b=c=d=\mathrm{e}^{\mathrm{i} e / N}}$, without differentiation. Note that we have sneaked in the factor $(a b / c d)^{(N-1) / 2}$ which becomes unity for the correlator $C$; our motivation for that import will be revealed below.

As a further consequence of the center-phase average, the generating function and the generalized correlator $\mathcal{C}$ depend on the four complex variables $a, b, c, d$ only through three independent combinations which may be chosen as

$$
a b=\mathrm{e}^{\mathrm{i} 2 e / N}, \quad \frac{c}{a}=\mathrm{e}^{\mathrm{i} \delta_{+} / N} \quad \frac{d}{b}=\mathrm{e}^{\mathrm{i} \delta_{-} / N} .
$$

While $\delta_{ \pm}$can be arbitrary complex, the variable $e$ is restricted by $\operatorname{Im} e>0$. Since we loose no physically important information by restricting ourselves to real $\delta_{ \pm}$that choice will be made without exception. Moreover, unless noted otherwise, we shall take the quasi-energy $e$ as real in the sense $\operatorname{Im} e \downarrow 0$.

We note in passing that the generating function $\mathcal{Z}$ defined above allows access to higher-order correlation functions, through higher derivatives w.r.t. to $c, d$ or to $\delta_{ \pm}$evaluated at $\delta_{ \pm}=0$. All those functions then appear, of course, with the single quasi-energy $e$ as the exclusive argument. There is thus physics in the generalized correlator $\mathcal{C}$ for $\delta_{ \pm}$near zero, for which reason we shall check for self-averaging there as well, not just at $\delta_{ \pm}=0$.

We can proceed to the Fourier transform of $\mathcal{C}\left(e, \delta_{+}, \delta_{-}\right)$with respect to $e$,

$$
\mathcal{K}\left(n, \delta_{+}, \delta_{-}\right)=\int_{0}^{\pi N} \frac{d e}{2 \pi} \mathcal{C}\left(e, \delta_{+}, \delta_{-}\right) \mathrm{e}^{-\mathrm{i} 2 e n / N}
$$

which we shall refer to as the generalized form factor since it reduces to the physical form factor for $\delta_{+}=\delta_{-}=0$.

For a given spectrum $\left\{\mathrm{e}^{-\mathrm{i} \phi_{\mu}}, \mu=1,2, \ldots N\right\}$ of the Floquet operator $U$, the generating function $\mathcal{Z}$ can be further evaluated by doing the center-phase average explicitly with the help of Cauchy's theorem. We may write the result as

$$
\mathcal{C}\left(e, \delta_{+}, \delta_{-}\right)=\frac{2}{N^{2}} \sum_{\mu, \nu=1}^{N} \frac{z \mathrm{e}^{-\mathrm{i} \Delta_{\mu \nu}}}{1-z \mathrm{e}^{-\mathrm{i} \Delta_{\mu \nu}}} f_{\mu}\left(\delta_{+}\right) f_{\nu}\left(-\delta_{-}\right), \quad \text { and } \quad \mathcal{K}\left(n, \delta_{+}, \delta_{-}\right)=\frac{1}{N} \sum_{\mu, \nu} \mathrm{e}^{-\mathrm{i} n \Delta_{\mu \nu}} f_{\mu}\left(\delta_{+}\right) f_{\nu}\left(-\delta_{-}\right)
$$


with

$$
z=\mathrm{e}^{\mathrm{i} 2 e / N}, \quad \Delta_{\mu \nu}=\phi_{\mu}-\phi_{\nu}, \quad \text { and } \quad f_{\mu}\left(\delta_{ \pm}\right)=\prod_{\nu(\neq \mu)} \frac{\sin \frac{\Delta_{\nu \mu}+\delta_{ \pm} / N}{2}}{\sin \frac{\Delta_{\nu \mu}}{2}} .
$$

We read off the period $\pi N$ for the $e$-dependence of $\mathcal{C}$ which allows to limit the range of $e$ to $[-\pi N / 2, \pi N / 2]$. Four properties of the auxiliary function $f_{\mu}\left(\delta_{ \pm}\right)$and their consequences for the generalized correlator and form factor are worth noting: (i) For real arguments $\delta_{ \pm}$the function $f_{\mu}\left(\delta_{ \pm}\right)$is real, whereupon $\operatorname{Re} \mathcal{C}$ exclusively parents the real correlator $R(e)=\operatorname{Re} C(e)$ through $\operatorname{Re} \frac{z \mathrm{e}^{\mathrm{i} \Delta_{\mu \nu}}}{1-z \mathrm{e}^{\mathrm{i}{ }_{\mu \nu}}}=-\frac{1}{2}+\pi \delta_{2 \pi}\left(\frac{2 e}{N}-\Delta_{\mu \nu}\right)$ - with the periodic delta functions arising for Im $e \downarrow 0$ - and analogously for the imaginary parts which contain principal-value $\frac{1}{e}$-singularities. That property is owed to the factor $\left(\frac{a b}{c d}\right)^{\frac{N-1}{2}}$ in the definition 3 of $\mathcal{C}$. A fine scale of variation in $e$ is worth being mentioned. Imagining the eigenvalue differences $\Delta_{\mu \nu}$ ordered as they increase from $-\pi$ to $\pi$ we can speak of a 'mean spacing' $\frac{2 \pi}{N^{2}}$ and the scale $\sim \frac{1}{N}$ for $e$. (ii) The product structure of $f_{\mu}$ reveals Fourier components $\mathrm{e}^{\mathrm{i} \nu \delta_{ \pm} / 2 N}$ for $\mathcal{C}$ and $\mathcal{K}$ with $\nu= \pm(N-1), \pm(N-3), \ldots$. Therefore, $\mathcal{C}$ and $\mathcal{K}$ are periodic in $\delta_{ \pm}$with period $2 \pi N$ for $N$ odd and $4 \pi N$ for $\mathrm{N}$ even; moreover, the finest scale of variation in $\delta_{ \pm}$is $2 \pi$. (iii) Due to $f_{\mu}(0)=1$ we again see the generalized correlator and form factor reduce to the physical ones for $\delta_{ \pm}=0$. (iv) For real $\delta_{+}=-\delta_{-}, \mathcal{K}$ becomes non-negative while $\mathcal{C}$ acquires a real (imaginary) part even (odd) in $e$. In particular, $R(e)=R(-e)$, and therefore we need to look at the real correlator $R(e)$ only in the interval $[0, \pi N / 2]$.

In preparation of the intended use of primitives of $\mathcal{C}$ we would like to point out that the explicit form of $\mathcal{C}$ given in (6) is a good starting point for integrating over $e$ (recall $z=\mathrm{e}^{\mathrm{i} 2 e / N}$ ), inasmuch as we are facing a slightly compacted form of the partial-fraction decomposition in terms of $\left(1-z \mathrm{e}^{-\mathrm{i} \Delta_{\mu \nu}}\right)^{-1}$. The first primitive $\int^{e} d e^{\prime} \mathcal{C}\left(e^{\prime}, \delta_{+}, \delta_{-}\right)$varies with the phase $\frac{e}{N}$ on the scale $\frac{1}{N^{2}}$, the latter measuring the typical distance between neighboring quasi-energy differences $\Delta_{\mu \nu}$. The real part of the first primitive is thus piecewise constant in $e$, with steps of height $\propto \frac{1}{N}$ and width $\propto \frac{1}{N^{2}}$. For $N \gg 1$ the real part of the first primitive will appear smooth on the scales $e \sim 1$ and $\int^{e} d e^{\prime} \mathcal{C} \sim 1$. On those scales, the imaginary part of $\int^{e} d e^{\prime} \mathcal{C} \sim 1$ also appears smooth, its inconspicuous logarithmic singularities notwithstanding. Given the effective smoothing, one expects variation of the first primitive of $\mathcal{C}$ on the scale $e \sim 1$ only.

\section{CUE AVERAGES}

Checking for self-averaging and universality means comparing spectral characteristics with their CUE averages. The generating function [6, 10, 12], $\left(\mathcal{Z}_{\mathrm{CUE}}-1\right) /[(a-c)(b-d)]=\left(1-(c d)^{N}\right) /[(1-a b)(1-c d)]$, yields,

$$
\mathcal{C}_{\mathrm{CUE}}\left(e, \delta_{+}, \delta_{-}\right)=\frac{\mathrm{ie}^{\mathrm{i} e}}{N^{2} \sin \frac{e}{N}} \quad \frac{\sin \left(e+\frac{\delta_{+}+\delta_{-}}{2}\right)}{\sin \left[\frac{1}{N}\left(e+\frac{\delta_{+}+\delta_{-}}{2}\right)\right]} \quad \Longrightarrow \quad \mathcal{C}_{\mathrm{CUE}}(e, \delta,-\delta)=C_{\mathrm{CUE}}(e)=\frac{\mathrm{e}^{\mathrm{i} 2 e}-1}{2 N^{2} \sin ^{2}(e / N)}
$$

The generalized correlator depends on the phases $\delta_{ \pm}$only through their sum $\delta_{+}+\delta_{-}$; that property is shared by the generalized form factor (5). The limit $N \rightarrow \infty$ with $\frac{e}{N} \rightarrow 0$ gives $C_{\mathrm{CUE}}(e) \rightarrow C_{\mathrm{GUE}}(e)=\frac{\mathrm{e}^{\mathrm{i} 2 e}-1}{2 e^{2}}$ and correspondingly $\mathcal{Z}_{\mathrm{GUE}}\left(e, \delta_{+}, \delta_{-}\right)$and $\mathcal{C}_{\mathrm{GUE}}\left(e, \delta_{+}, \delta_{-}\right)$. The two determinants in the denominator of (1) have their zeros along the $\phi$-axis shifted by $2 e$ whereas those in the numerator have relative shift $2 e+\delta_{+}+\delta_{-}$. It is these two shifts which are the arguments in the factors of the CUE generalized correlator.

\section{SMOOTHING BY INTEGRATION}

The first primitive of the real correlator $R=\operatorname{Re} C$ can be defined as $R^{(1)}(e)=\int_{e}^{N \pi / 2} d e^{\prime} R\left(e^{\prime}\right)$ with the reference point at the right border of the principal interval of $e$. It is close to zero for all $e$ comparable to $N$; when $e$ tends to +0 the first primitive of the physical correlator with $\delta_{+}=\delta_{-}=0$ tends to the universal limit $-\pi / 2$ for any spectrum. The first primitive experiences a discontinuity at $e=0$ due to the diagonal terms in the sum (6). When $\delta_{ \pm}=0$ and, generally, when $\delta_{+}=-\delta_{-}$we have $R^{(1)}(0)=0$ such that the first primitives with the reference point at zero and at $N \pi / 2$ coincide, up to the sign. These properties can be understood considering that every physical pair of eigenphases makes one and one only contribution to $R^{(1)}(0)$.

The first primitive is known to be self-averaging and faithful to random-matrix theory for fully chaotic dynamics [5], as illustrated for the kicked top in Fig. 1, the phase $\frac{e}{N}$ is reckoned there in units of the mean level spacing (ms) $\frac{2 \pi}{N}$, such that the mean level spacing in terms of the quasi-energy $e$ is $2 \pi$. However, self-averaging and universality reign only for $e$ in a range of a few $\mathrm{ms}$, in the region of noticeable level-level correlations. - Outside that region, in 
particular for $e \sim N, R^{(1)}(e)$ fluctuates around zero without systematic $e$-dependence, see Fig. 2. the black ribbon of constant width consists of irregular fluctuations; the inset shows a blow-up. The noise amplitude turns out to decay as $1 / \sqrt{N}$ with $N \rightarrow \infty$ (accuracy better than $2 \%$ for absolute deviation from mean, over two decades of $N$ ). Both the CUE average, $R_{\mathrm{CUE}}^{(1)}(e) \propto N^{-1}$ and the difference between the CUE and GUE correlators are overwhelmed by the noise. It follows that a single spectrum does not allow to distinguish the infinite- $N$ GUE correlator from the finite- $N$ CUE one, the periodicity of the latter apart. - The overall behavior of $R^{(1)}(e)$ for individual kicked tops is consistent with the 'ergodicity' of the correlator within the CUE [5, 7], to be looked at more closely in Sect. VI]

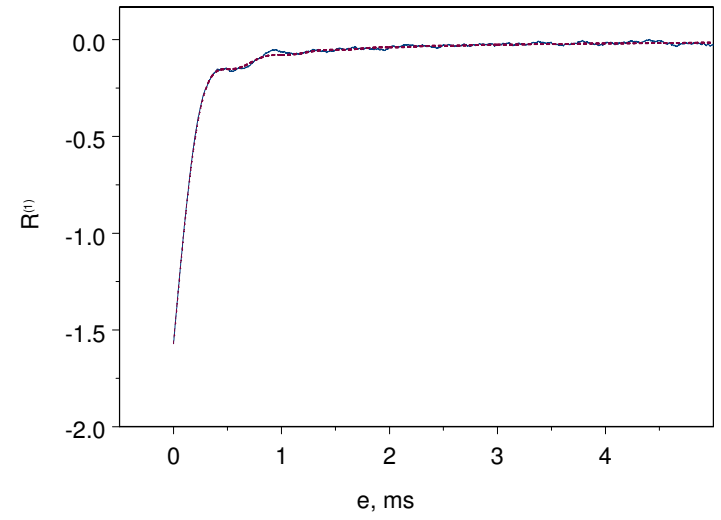

FIG. 1: Kicked top, $j=9600$. First primitive $R^{(1)}(e)$ with $\delta=0$ practically coincides with RMT (dashed)

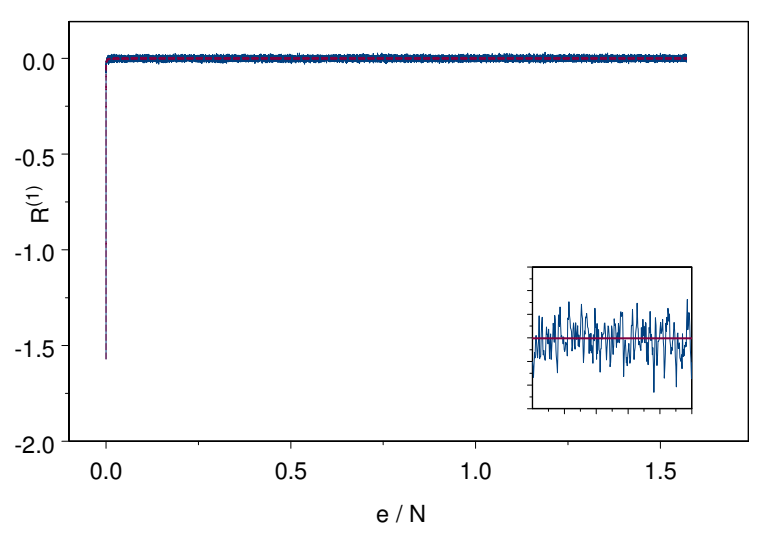

FIG. 2: Same but for large $e$. Only noise remains once $e$ exceeds a few ms. Blowup of noise stretch in inset

As mentioned above, the first primitive appears smooth on the physically most interesting scales. On the other hand, if one wants to strictly ban all singularities one may focus on the second primitive of $\mathcal{C}$ which is continuous in the phase $e$. In that vein we define

$$
\mathcal{C}^{(2)}\left(e, \delta_{+}, \delta_{-}\right)=\int_{0}^{e} d e^{\prime} \int_{\frac{N \pi}{2}}^{e^{\prime}} d e^{\prime \prime} \mathcal{C}\left(e^{\prime \prime}, \delta_{+}, \delta_{-}\right) \quad \text { and } \quad \mathcal{K}^{(2)}\left(n, \delta_{+}, \delta_{-}\right)=\sum_{n^{\prime}=1}^{n} \sum_{n^{\prime \prime}=1}^{n^{\prime}} \mathcal{K}\left(n^{\prime \prime}, \delta_{+}, \delta_{-}\right)
$$

Our numerical studies of the second primitives reveal self-averaging and fidelity to RMT only for $\left|\delta_{ \pm}\right|$at most of order unity and for $e$ within the windows of correlation decay (around $|e|=0 \bmod N \pi)$ already met with in $R^{(1)}(e)$. The self-averaging of $R^{(2)}, K^{(2)}$, together with the mentioned limitations, is revealed in Figs. 3 . 4, and 5 .

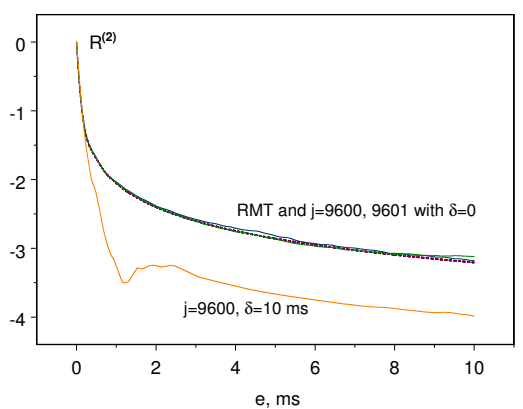

FIG. 3: self-averaging $\operatorname{Re} \mathcal{C}^{(2)}$ of kicked top for $e \ll N, \delta=0$. Violation of universality for $\delta=10 \mathrm{~ms}$

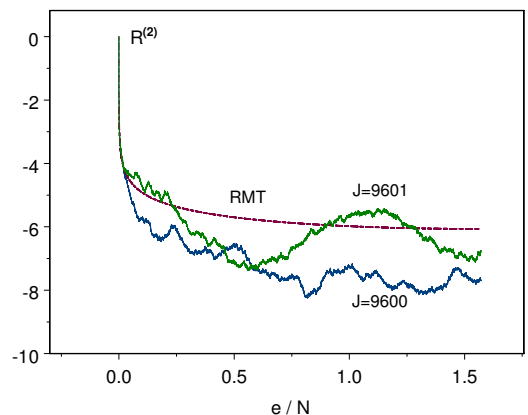

FIG. 4: $R^{(2)}$ noisy and non-universal for large $e$

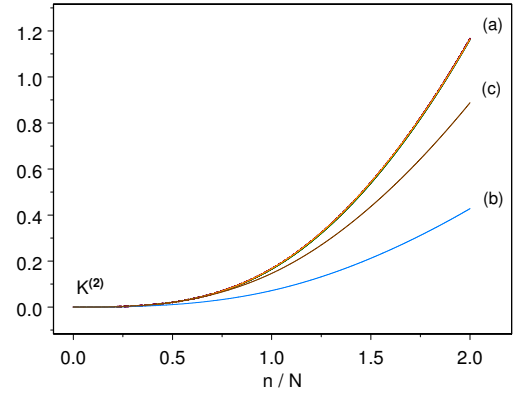

FIG. 5: (a) self-averaging $\mathcal{K}^{(2)}$ of kicked top for $\delta=0$ and $\delta=1 \mathrm{~ms}$. $(\mathrm{b}, \mathrm{c})$ No universality for $\delta=10 \mathrm{~ms}$

In particular, Fig. 3 depicts a narrow bundle of three curves for the second primitive of the real correlator $R^{(2)}(e)$, one representing the CUE average and the other two pertaining to the kicked top with $j=9600$ and 9601 in the 
range of $e$ between zero and $10 \mathrm{~ms}$. Throughout that range, the three curves differ from one another by but hardly noticeable amounts. It is to be noted that for $e$ equaling $5 \mathrm{~ms}$ the CUE correlator $R_{\mathrm{CUE}}(e)$ has decayed to a practically negligible level $\sim \frac{1}{(10 \pi)^{2}}$. A fourth curve in Fig. 3 pertains to the second primitive of the real part of the generalized correlator $\mathcal{C}\left(e, \delta_{ \pm}\right)$with $\delta_{+}=-\delta_{-}=\delta$ equaling 10 mean spacings. That curve strongly deviates from the CUE prediction (which as already mentioned does not depend on $\delta$ ) and signals non-self-averaging behavior.

For larger values of $e$ the relative differences grow and signal non-universality, see Fig. 4 However, that growth is of no importance for two reasons. First, outside the phase window of correlation decay (and revival; note the periodicity with period $\pi N$ ) only weak noise remains for $R^{(1)}(e)$. Second, the second primitive grossly exaggerates all large- $e$ structures, turning the $\frac{1}{e^{2}}$ decay of $R_{\mathrm{CUE}}(e)$ into a logarithmic one.

The same salient message is signaled in Fig. 5 for the second primitive of the form factor as a function of the scaled discrete time $\frac{n}{N}$. Three curves (a), unresolved from one another, refer to the CUE and the kicked top for $j=9600$ with $\delta_{+}=-\delta_{-}=\delta$ equal to zero and a single mean spacing indicate excellent self-averaging and universality. Two further curves pertain to the kicked top with $\delta=10 \mathrm{~ms}$, one (b) for $j=9600$ and the other (c) for $j=9601$. Universality would require both curves (b,c) to coincide with the triple (a) and is grossly violated. The noisy small-time behavior of $\mathcal{K}^{(2)}(n, \delta,-\delta)$, invisible on the scale of Fig. 5 , corresponds to the large-e noise in $\mathcal{C}^{(2)}$.

\section{SMOOTHING BY COMPLEX QUASI-ENERGY}

In Fig. 6 we show the real correlator $R(e)=\operatorname{Re} C(e+\mathrm{i} \eta)$ where $\eta$ is a small imaginary addition to the real quasienergy $e$. To smooth away the delta peaks arising for vanishing $\eta$ that imaginary part must be larger than the finest scale of variation in $e$, seen to be $\sim \frac{1}{N}$ at the end of Sect. II. On the other hand, we want to keep $\frac{2 \eta}{N}$ smaller than the mean spacing $\frac{2 \pi}{N}$ of the eigenvalues of $U$, in order not to tamper with the $e$ dependence on that latter scale. These restrictions are respected in Fig. 6. Just as for smoothing by integration we see excellent agreement with the CUE average within the principal window of correlation decay but noise dominance outside: for $\frac{e}{N}$ not small the CUE average scales as $\frac{1}{N^{2}}$ while the standard deviation is found to decay only like $\frac{1}{\sqrt{N}}$ as $N$ grows at fixed $\eta$.

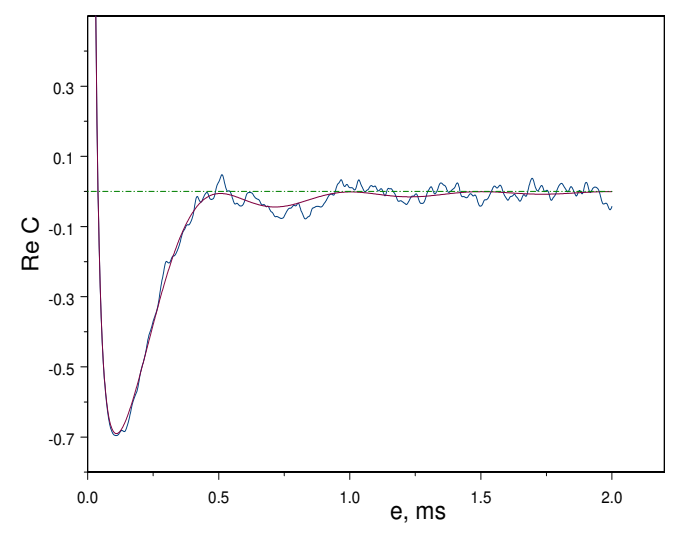

FIG. 6: Physical correlator $R=\operatorname{Re} C$ of a kicked top spectrum, $j=9600$. Regularization by imaginary part Im $e=0.01$ ms. Smooth curve shows CUE prediction.

\section{CUE FLUCTUATIONS}

In order to better understand the noise in single-top correlators and the systematic non-self-averaging in the generalized correlator, we here look at fluctuations of the single- $U$ correlator throughout the CUE. We start with some numerical findings and shall afterwards proceed to discussing an explicit analytic result for the CUE variance. 


\section{A. Numerical results}

Fig. 7 further conveys the absence of self-averaging and universality in the generalized correlator (and thus the generating function) outside the physically relevant range of its variables. The 3D plot in Fig. 7 shows the mean absolute deviation $\left\langle\left|\operatorname{Re}^{(2)}-\operatorname{Re}_{\mathrm{CUE}}^{(2)}\right|\right\rangle$, the mean $\langle\cdot\rangle$ taken over 13937 spectra of $201 \times 201$ matrices randomly drawn from the CUE (with the algorithm described in [13]), as a function of $\delta_{+}$and $\delta_{-}$with $e=1 \mathrm{~ms}$; the range captured for $\delta_{ \pm}$is chosen as $20 \mathrm{~ms}$, symmetric about zero. The line $\delta_{+}+\delta_{-}=0$ is obviously distinguished: Thereon, the mean absolute deviation is small only in a narrow interval near $\delta_{+}=\delta_{-}=0$ while outside a high ridge arises and signals large fluctuations of $R^{(2)}$ within the CUE; a single unitary matrix, either for a kicked top or drawn at random from the CUE, would entail even stronger and noisier absolute deviation and thus reveal non-self-averaging. Perpendicular to the line $\delta_{+}+\delta_{-}=0$ there is less drama: The mean absolute deviation undergoes oscillations, roughly with the finest scale of variation allowed by the Fourier series for $f_{\mu}\left(\delta_{ \pm}\right)$, and decay with increasing $\left|\delta_{+}-\delta_{-}\right|$. Even though less flagrant and not obvious from Fig. 7. non-self-averaging is also incurred along the line $\delta_{+}-\delta_{-}=0$ with increasing distance from the center $\delta_{+}=\delta_{-}=0$; already a few mean spacings beyond the center, relative mean absolute deviations of order unity become typical.

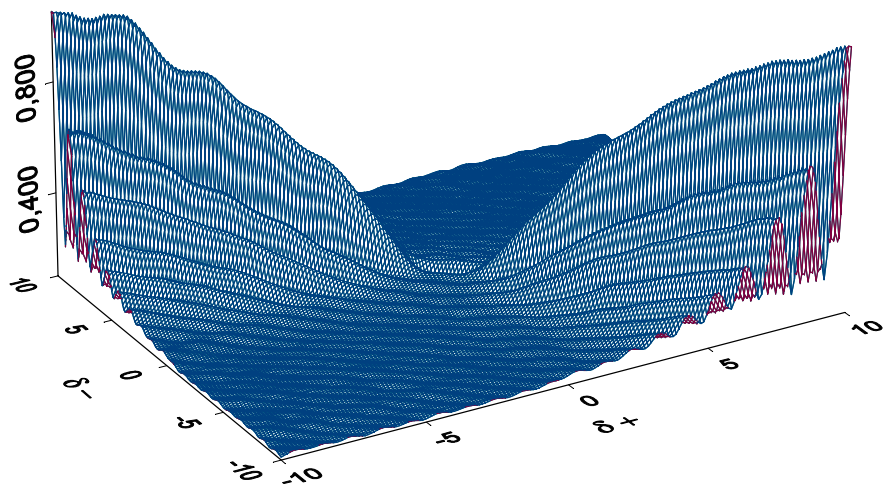

FIG. 7: Absolute deviation $\left\langle\left|\operatorname{Re}^{(2)}-\operatorname{Re}_{C U E}^{(2)}\right|\right\rangle$ of the second primitive of the generalized correlator from its CUE expectation value at fixed $e=1 \mathrm{~ms}$. The result is an average over 13937 spectra of $201 \times 201$ random CUE matrices.

When accumulating the many spectra making up Fig. 7 we have observed slow convergence, the slowness due to 'rare extreme events'; a single odd spectrum can significantly alter the partial average previously accumulated. We suspect that such extreme events are spectra with exceptionally close nearest-neighbor pairs of levels; for such spectra the coefficients $f_{\mu}$ in the spectral representation (6) become almost singular and thus give rise to huge deviations from the universal limit and flagrant absence of self-averaging as soon as $\left|\delta_{+}-\delta_{-}\right|$exceeds a few mean spacings. That suspicion is nourished by an analogous investigation of the circular orthogonal ensemble where weaker level repulsion makes this scenario more probable; as demonstrated in Section VIIIB of the Appendix we found even slower convergence and orders-of-magnitude larger variance of the generalized correlator with the same $\delta_{ \pm} \neq 0$ than for the CUE. Similar calculations for the circular symplectic ensemble with the level repulsion even stronger than in CUE, demonstrated opposite tendencies.

\section{B. Analytic results}

All of the foregoing numerical results are supported and in part generalized by the CUE variance of the correlator smoothed by either an imaginary part of the quasi-energy $e$ or by integration. That variance can be extracted from the CUE-averaged combination of eight spectral determinants $\left\langle\frac{D D D D}{D D D D}\right\rangle_{\mathrm{CUE}}$ where each $D$ has its own quasi-energy argument [10; the result has yet to be averaged over the relative central phase of the two $\frac{D D}{D D}$ ratios. We shall here 
discuss the final expression for the variance which generalizes Pandey's ergodicity of the correlator within the CUE [5, 7]. For details see Appendix, Section VIII A.

Complex quasi-energy: We first turn to the generalized correlator smoothed by an imaginary part $\eta$ added to the real quasi-energy $e$. The pair $\delta_{ \pm}$will be left real and even restricted as $\delta_{+}=-\delta_{-}=\delta$. The latter special case deserves special attention due to the strong fluctuations at large $\delta$ seen in Fig. 7 while the CUE average is $\delta$-independent. The variance $\operatorname{Var}_{\mathrm{CUE}}(\operatorname{Re} \mathcal{C}(e+\mathrm{i} \eta, \delta))$ is given by a somewhat cumbersome expression; we show only the leading term of its expansion in powers of $\eta$,

$$
\operatorname{Var}_{\mathrm{CUE}}(\operatorname{Re} \mathcal{C}(e+\mathrm{i} \eta, \delta)) \sim \frac{1}{2 N \eta}\left(1-\frac{\sin ^{2} \frac{\delta}{2 N}}{\sin ^{2} \frac{e}{N}}\right)^{2}\left(1-\frac{\sin ^{2} e}{N^{2} \sin ^{2} \frac{e}{N}}\right)(1+\eta f(e, N)+\ldots)
$$

with $f(e, N)$ finite for all $e$ and $N \rightarrow \infty$ [18. In particular, the variance of the physical correlator, $\operatorname{Var}_{\mathrm{CUE}} \operatorname{Re}(C(e+$ $\mathrm{i} \eta)$ ), arises for $\delta=0$ and is seen to be $\propto \frac{1}{N \eta}$. For it to become small for large $N$ the smoothing imaginary part $\eta$ must, as already argued in Sect. V, be larger than the minimum scale of variation in $e$, that is $\frac{1}{N}$, and small compared to the mean spacing, that is $2 \pi$ in the units used for $e$. These restrictions are respected for $\eta \propto N^{-\alpha}$ with $0<\alpha<1$ and then the variance of the physical correlator is $\propto N^{-1+\alpha}$, small indeed and suggesting self-averaging of the correlator. On the other hand, we find confirmed the numerical finding that outside the windows of correlation decay and revival where $\frac{e}{N}$ is of order unity, the fluctuations overwhelm the mean; in fact the standard deviation exceeds the mean already at much smaller energies, namely when $e \gtrsim \sqrt[4]{\eta N}$.

Even more drastic is the non-self-averaging of the generalized correlator when $e$ ranges within the windows of correlation decay and revival while $\delta \sim N^{\beta}, \quad 0<\beta \leq 1$; the variance then becomes $\sim N^{4 \beta-1+\alpha}$.

A bit surprising is the smallness of the generalized correlator variance $\left(\sim \frac{1}{N \eta}=N^{-1+\alpha}\right)$ when both $e$ and $\delta$ are of order $N$, but there again fluctuations overwhelm the mean. At any rate, the region $\delta \gg 1$ is devoid of physical interest.

Primitives: Proceeding to smoothing by integration we note the variance of the first primitive $R^{(1)}(e)$ at $\operatorname{Im} e \downarrow 0$,

$$
\begin{aligned}
\operatorname{Var}_{\mathrm{CUE}} R^{(1)}(e) & =\int_{0}^{e} d y\left[\frac{\sin 4 y}{N^{3} \sin ^{2} \frac{2 y}{N}} \ln \frac{\sin ^{2} \frac{e-2 y}{N}}{\sin ^{2} \frac{e}{N}}+\frac{\pi}{N}\left(1-\frac{\sin ^{2} y}{N^{2} \sin ^{2} \frac{y}{N}}\right)\right. \\
& \left.+\frac{(e-y)\left(4 N \cos 4 y-2 \cot \frac{2 y}{N} \sin 4 y\right)}{N^{4} \sin ^{2} \frac{2 y}{N}}-\frac{1}{N y} \ln \frac{(e-2 y)^{2}}{e^{2}}\right] .
\end{aligned}
$$

The variance is exactly zero at $e=0$ and $e=N \pi / 2$ which is understandable since $R^{(1)}(e)$ of any spectrum has the same value at these points. Away from the end points the variance steeply rises to approximately $1 / N$. It is not difficult to extract the large- $N$ asymptotics within the principal window of correlation decay and revival where $\frac{e}{N} \ll 1$

$$
\begin{aligned}
\operatorname{Var}_{\mathrm{CUE}} R^{(1)}(e) & \sim \frac{1}{N}\left[1-\frac{1}{2} \cos 4 e-\frac{\sin 4 e}{8 e}-2 e \operatorname{Si} 4 e\right. \\
& \left.+\pi\left(e+\frac{\sin ^{2} e}{e}-\operatorname{Si} 2 e\right)+\int_{0}^{e} \frac{(\sin 4 y-4 y) \log \left|1-\frac{2 y}{e}\right|}{2 y} d y\right]
\end{aligned}
$$

here Si $x=\int_{0}^{x} \frac{\sin t}{t} d t$. In Fig. 8, both the exact and the asymptotic form of $\operatorname{Var}_{\mathrm{CUE}} R^{(1)}(e)$ are depicted for $N=11$. Both curves practically agree, except for $e$ in the immediate neighborhood of the half period, where the large- $N$ asymptotics cannot fall back to 0 . The close agreement of the two curves in the figure is in fact amazing since the large- $N$ asymptotics is derived only for small $\frac{e}{N}$. The scaling with $N$, $\operatorname{Var}_{\mathrm{CUE}} R^{(1)}(e) \sim \frac{1}{N}$, is manifest in the figure.

The overwhelming of the mean by fluctuations for large $e, \frac{e}{N} \sim 1$, is found again, here for the first primitive $R^{(1)}(e)$ such that the ratio of standard deviation and mean is of the order $\sqrt{N}$.

To characterize the CUE fluctuations of the generalized correlator we finally comment on the variance of Re $\mathcal{C}^{(1)}(e, \delta)$ where again $\delta=\delta_{+}=-\delta_{-}$. We sketch the explicit result only for the $e$-window of correlation decay and revival, $\frac{e}{N} \ll 1$

$$
\operatorname{VarRe} C^{(1)}(e, \delta) \approx\left(\operatorname{Var} R^{(1)}(e)\right)\left[1+N^{2} \sin ^{2} \frac{\delta}{2 N} F_{1}(e)+N^{4} \sin ^{4} \frac{\delta}{2 N} F_{2}(e)\right]
$$

where $F_{1}, F_{2}$ are some $N$-independent functions of $e$. The most important conclusion is negligibility of fluctuations in the same sense as for $\delta=0$, as long as $\delta$ remains of order unity. The extreme case of $\delta \sim N$ has $\operatorname{VarRe} C^{(1)}(e, \delta) \sim N^{3}$ 


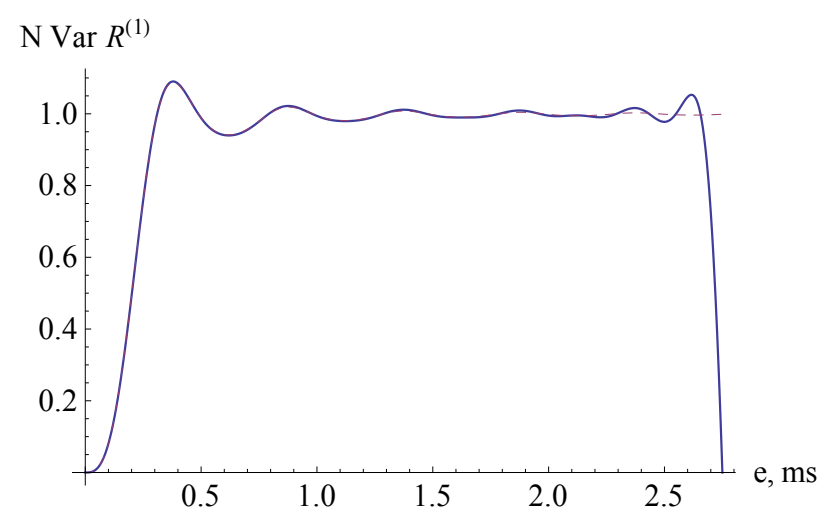

FIG. 8: Variance of the first primitive of the physical correlator with $N=11$, exact (full line) and large- $N$ asymptotics (dashed)

and thus no self-averaging. Even the less excessive growth $\delta \sim N^{\beta}$ with $\beta<1$ gives $\operatorname{VarRe} C^{(1)}(e, \delta) \sim N^{4 \beta-1}$ and thus loss of self-averaging for $\delta>\frac{1}{4}$. Indeed, in our numerical calculations the largest $N$ was of the order $10^{4}$ with $N^{1 / 4} \sim 10$; the break-up of self-averaging could thus be expected (and was observed) at $\delta \gtrsim 2 \pi=1 \mathrm{~ms}$.

\section{DISCUSSION AND OUTLOOK}

We have found fidelity to RMT of the first and second primitives of spectral form factor $K(n)$ and real two-point correlator $R(e)$ for an individual kicked top in periodically (period $\propto N$ ) repeated windows of correlation decay and revival for the quasi-energy variable $e$. In between these windows correlations are so weak as to be negligible for large $N$. System specific noise in the $e$-dependence is found to have the order $\frac{1}{\sqrt{N}}$ and thus to be negligible as well. Interestingly, however, the noise overwhelms the CUE average of the correlator in between the windows of correlation decay and revival. In fact the noise is strong enough to preclude distinguishability of the CUE and GUE prediction for a single spectrum. The same behavior is found when the correlator is smoothed by an imaginary addition i $\eta$ to the real quasi-energy $e$, provided $\eta$ is (i) large enough to 'iron out' the singularities in the $\eta \downarrow 0$ correlator and (ii) small enough to not noticeably attenuate $R(e)$. On the other hand, we find the underlying generating function $\mathcal{Z}\left(e, \delta_{+}, \delta_{-}\right)$ self-averaging and universal only under the additional restriction of $\left|\delta_{ \pm}\right|$no larger than a few mean spacings.

Our results for the CUE variance of the correlator $R(e)$, the latter smoothed by suitable $\eta$ or integration, mean that in the large- $N$ limit any CUE matrix can with overwhelming probability (for $N \rightarrow \infty$ with probability one) be expected to have a smoothed correlator equaling the CUE average. Likewise, by working out the smoothed correlator for a single top (or any other fully chaotic map) with large $N$, one has overwhelming probability to get the CUE average. Exceptions are possible but will in practice not be met with.

We would like to comment on the status of previous semiclassical work involving quadruples of classical pseudoorbits from narrowly packed bunches. Such work has resulted in universality of $\mathcal{Z}\left(e, \delta_{+}, \delta_{-}\right)$, without restriction for the independent variables and without manifest necessity of any ensemble average 4,6 . That periodic-orbit approach involves a 'perturbation series' for $\mathcal{Z}$. The original work on autonomous flows 4 relied on an imaginary part $\eta$ of the quasi-energy variable much larger than a mean spacing; return to real quasi-energy was possible only after summing up the series. The later extension to Floquet maps [6] could make do with 'infinitesimal' $\eta$. Our present investigation reveals limits within which $\eta$ must lie for the semiclassical periodic-orbit expansions to be applicable to individual Floquet maps, at least for the correlator $R(e)$ in the limit $N \rightarrow \infty$.

On the other hand, we have found here that one may stick to real quasi-energies (in the sense $\eta \downarrow 0$ ) if one smoothes by going to the first primitive $R^{(1)}(e)$; self-averaging then takes place for $N \rightarrow \infty$ and even for large finite $N$ apart from negligible noise. The semiclassical periodic-orbit expansions can be done under the protection of that smoothing, and then smoothing by integration also 'has the right' to give the single-dynamics $R^{(1)}(e)$ equal to the CUE average.

Inasmuch as periodic orbits yield the CUE average of the full generating function $\mathcal{Z}\left(e, \delta_{+}, \delta_{-}\right)$it appears that 'too much is proven' since we now know that a single spectrum comes with gross violations of self-averaging for large $\delta_{ \pm}$. One does not need to worry too much since large $\delta_{ \pm}$do not harbor any physically relevant information. But with the correlator (or its primitive) satisfactorily treated one can live with subjecting the periodic-orbit expansion to a suitable further average to justify the RMT result for $\mathcal{Z}$. We have checked that an average over $N \propto \frac{1}{\hbar}$ leaving unchanged the classical limit does the job for our kicked top. Averages over small intervals of classical control parameters (small in 
the sense of shrinking to zero length for $\hbar \rightarrow 0$ ) have been shown to work as well [11.

We conclude with a speculative outlook. As already stated above and illustrated in Fig. 4, the second primitive enhances large-e structures. Exploiting that property we have averaged the primitive $R^{(2)}(e)$ for single kicked-top spectra over a range $[j, j+\Delta j]$ with $1 \ll \Delta j \ll j$. That average did away with noise at large $e \propto N=2 j+1$ but left oscillations in $e$, around the CUE average $R_{\mathrm{CUE}}^{(2)}(e)$. On the other hand, when doing the same with matrices randomly drawn from the CUE we found no oscillations. Further investigation must reveal whether the oscillations found for the top are due to Ehrenfest-time effects [14, 16, short orbits, or some other effect.

We thank Sven Gnutzmann for discussions and gratefully acknowledge support by the Sonderforschungsbereich SFBTR12 "Symmetries and universality in mesoscopic systems" of the Deutsche Forschungsgemeinschaft.

\section{APPENDIX}

\section{A. RMT predictions for covariance and variance of the generating functions, generalized correlators and correlator primitives}

The covariance of two generating functions $Z\left(a_{i}, b_{i}, c_{i}, d_{i}\right) \equiv Z(i), \quad i=1,2$, can be defined as

$$
\operatorname{Cov}\{Z(1), Z(2)\}=\langle Z(1) Z(2)\rangle-\langle Z(1)\rangle\langle Z(2)\rangle
$$

where $\langle\ldots\rangle$ stands for the CUE average; the second summand in the right -hand side is a product of the well-known CUE generating functions. The first summand is formally a double integral over the central phases $\phi_{1,2}$ of the two $\mathrm{DD} / \mathrm{DD}$ ratios, but in view of the CUE averaging only integration over the relative central phase is needed,

$$
\begin{aligned}
\langle Z(1) Z(2)\rangle= & \int_{0}^{2 \pi} \frac{d \phi}{2 \pi}\left\langle\frac{\operatorname{det}\left(1-c_{1} \mathrm{e}^{\mathrm{i} \phi} U\right) \operatorname{det}\left\langle 1-d_{1} \mathrm{e}^{-\mathrm{i} \phi} U^{\dagger}\right\rangle}{\operatorname{det}\left(1-a_{1} \mathrm{e}^{\mathrm{i} \phi} U\right) \operatorname{det}\left(1-b_{1} \mathrm{e}^{-\mathrm{i} \phi} U^{\dagger}\right)}\right. \\
& \left.\times \frac{\operatorname{det}\left(1-c_{2} U\right) \operatorname{det}\left\langle 1-d_{2} U^{\dagger}\right\rangle}{\operatorname{det}\left(1-a_{2} U\right) \operatorname{det}\left(1-b_{2} U^{\dagger}\right)}\right\rangle .
\end{aligned}
$$

The integrand can be imported from [10, Eq.(1.1); integration over $\phi$ is straightforward. The result can be reformulated as the covariance of two generalized correlators (3). We present the final expression only for the special case when in both correlators $\delta_{+}=-\delta_{-}=\delta$; denoting $z_{k}=\mathrm{e}^{12 e_{k} / / N}, k=1,2$, we have then,

$$
\operatorname{Cov}\left\{C\left(e_{1}, \delta\right), C\left(e_{2}, \delta\right)\right\}=\frac{4}{N^{4}} A\left(z_{1}, z_{2}, \delta\right) B\left(z_{1}, z_{2}\right),
$$

with

$$
\begin{aligned}
A\left(z_{1}, z_{2}, \delta\right)= & \frac{z_{1} z_{2}}{\left(z_{1} z_{2}-1\right)^{2}} \prod_{k=1,2} \frac{\left(z_{k} \mathrm{e}^{\mathrm{i} \delta / N}-1\right)\left(z_{k} \mathrm{e}^{-\mathrm{i} \delta / N}-1\right)}{\left(z_{k}-1\right)^{2}} \\
B\left(z_{1}, z_{2}\right)= & -\frac{z_{1} z_{2}+1}{z_{1} z_{2}-1}+\frac{z_{1} z_{2}-1}{z_{1}-z_{2}}\left[\frac{z_{2}^{N}\left(z_{1}+1\right)}{z_{1}-1}-\frac{z_{1}^{N}\left(z_{2}+1\right)}{z_{2}-1}\right] \\
& +z_{2}^{N} z_{1}^{N}\left(\frac{2}{z_{1}-1}+\frac{2}{z_{2}-1}+\frac{6}{z_{1} z_{2}-1}+3-2 N\right)
\end{aligned}
$$

Assuming $\delta$ real we have for the complex conjugated correlator, $\bar{C}(e, \delta)=C(\bar{e}, \delta)$. Therefore the covariance of the correlator real parts can be written,

$$
\begin{aligned}
& \operatorname{Cov}\left\{\operatorname{Re} C\left(e_{1}, \delta\right), \operatorname{Re} C\left(e_{2}, \delta\right)\right\} \\
= & \frac{1}{2} \operatorname{Re}\left[\operatorname{Cov}\left\{C\left(e_{1}, \delta\right), C\left(e_{2}, \delta\right)\right\}+\operatorname{Cov}\left\{C\left(e_{1}, \delta\right), C\left(\bar{e}_{2}, \delta\right)\right\}\right]
\end{aligned}
$$

The variance of the correlator real part is obtained from 15 in the limit $e_{1}, e_{2} \rightarrow e$,

$$
\begin{aligned}
\operatorname{Var}_{\mathrm{CUE}} \operatorname{Re} C(e, \delta) & \equiv\left\langle(\operatorname{Re} C(e, \delta))^{2}\right\rangle-\langle\operatorname{Re} C(e, \delta)\rangle^{2} \\
& =\frac{1}{2} \operatorname{Re}[A(z, z, \delta) B(z, z)+A(z, \bar{z}, \delta) B(z, \bar{z})] ;
\end{aligned}
$$


note that

$$
\begin{aligned}
B(z, z)= & -\frac{z^{2}+1}{z^{2}-1}-z^{N-1} \frac{(z+1)\left[2 z+N\left(z^{2}-1\right)\right]}{z-1} \\
& +z^{2 N}\left(\frac{4}{z-1}+\frac{6}{z^{2}-1}+3-2 N\right) .
\end{aligned}
$$

In the limit Im $e_{1,2} \downarrow 0$ the covariance of the physical correlators $R\left(e_{1,2}\right)=\operatorname{Re} C\left(e_{1,2}+\mathrm{i} 0,0\right)$ becomes a sum of two expressions differing by the replacement $e_{2} \rightarrow-e_{2}$,

$$
\begin{aligned}
\operatorname{Cov}\left\{R\left(e_{1}\right), R\left(e_{2}\right)\right\}= & {\left[\delta\left(e_{1}-e_{2}\right) \frac{\pi}{N}\left(1-\frac{\sin ^{2} e_{1}}{N^{2} \sin ^{2} \frac{e_{1}}{N}}\right)+\frac{\cos 2\left(e_{1}-e_{2}\right)}{N^{3} \sin ^{2} \frac{e_{1}-e_{2}}{N}}\right.} \\
& \left.+\frac{\sin 2\left(e_{1}-e_{2}\right)\left(1+\cos \frac{2 e_{1}}{N}+\cos \frac{2 e_{2}}{N}-3 \cos \frac{2\left(e_{1}-e_{2}\right)}{N}\right)}{8 N^{4} \sin ^{3} \frac{e_{1}-e_{2}}{N} \sin \frac{e_{1}}{N} \sin \frac{e_{2}}{N}}\right] \\
& +\left[e_{2} \rightarrow-e_{2}\right] ;
\end{aligned}
$$

the variance becomes infinite as signalled by $\delta\left(e_{1}-e_{2}\right)$.

The covariance of the real parts of two generalized correlators with $e_{1,2}>0$ is proportional to (17),

$$
\begin{aligned}
& \operatorname{Cov}\left\{\operatorname{Re} C\left(e_{1}, \delta\right), \operatorname{Re} C\left(e_{2}, \delta\right)\right\} \\
= & \left(1-\frac{\sin ^{2} \frac{\delta}{2 N}}{\sin ^{2} \frac{e_{1}}{N}}\right)\left(1-\frac{\sin ^{2} \frac{\delta}{2 N}}{\sin ^{2} \frac{e_{2}}{N}}\right) \operatorname{Cov}\left\{R\left(e_{1}\right), R\left(e_{2}\right)\right\} ;
\end{aligned}
$$

in addition, it contains singular terms $\propto \delta\left(e_{1}\right), \delta\left(e_{2}\right)$ which we do not write out.

The variance of the real part of the correlator first primitive $C^{(1)}(e, \delta)=\int_{e}^{N \pi / 2} d e^{\prime} C\left(e^{\prime}, \delta\right)$ is obtained from the covariance (15) by double integration,

$$
\begin{aligned}
& \operatorname{Var}\left\{\operatorname{Re} C^{(1)}(e, \delta)\right\} \equiv\left\langle\operatorname{Re} C^{(1)}(e, \delta) \operatorname{Re} C^{(1)}(e, \delta)\right\rangle-\left\langle\operatorname{Re} C^{(1)}(e, \delta)\right\rangle^{2} \\
= & \int_{e}^{N \pi / 2} \int_{e}^{N \pi / 2} \operatorname{Cov}\left\{\operatorname{Re} C\left(e_{1}, \delta\right), \operatorname{Re} C\left(e_{2}, \delta\right)\right\} d e_{1} d e_{2} .
\end{aligned}
$$

In view of the relation $\int_{0}^{e} \operatorname{Re} C\left(e^{\prime}, \delta\right) d e^{\prime}=-\int_{e}^{N \pi / 2} \operatorname{Re} C\left(e^{\prime}, \delta\right) d e^{\prime}$ the integration area can also be the square $[0 \leq$ $\left.e_{1,2} \leq e\right]$; singularities of the covariance at $e_{1,2}=0$ omitted in $(18)$ have then to be taken into account.

\section{B. Variance of generalized correlator in CUE, COE and CSE: numerical simulation}

As we pointed out in the main text, the variance of the generalized correlator as function of $\delta$ must be drastically different for the three Dyson ensembles because of different level repulsion: Compared with CUE, $\beta=2$, the correlator fluctuations at large $\delta$ must be more pronounced in COE, $\beta=1$, and less so in CSE, $\beta=4$. We checked these predictions computing the standard deviation as function of $\delta$ in the ensembles of $10^{7} \mathrm{CUE}, 10^{6} \mathrm{CSE}$ and $10^{8} \mathrm{COE}$ matrices; the CSE matrices were generated by the method suggested in [17. The chosen number of matrices in each ensemble depended on the rate of convergence which was the slowest in COE and the fastest in CSE. The real part of energy was fixed at $e_{0}=1 \mathrm{~ms}$ and its imaginary part at $\eta=0.05 \mathrm{~ms}$; the matrix size was $N=21$. The result is shown by the full curves in Fig. 9 .

The deviation in the three ensembles was close at $\delta=0$, i. e., for the physical correlators. On the other hand, at large $\delta$ the mean deviations of the numerical COE and CSE correlator differed, in opposite directions, from its CUE counterpart by up to three orders of magnitude; this can be interpreted as confirmation of our hypothesis on the role of the level repulsion.

The dashed curve in Fig. 9 depicts the theoretical CUE deviation following from the exact Eq. 16;; good agreement with the numerics in a wide range is obvious.

Note the sharp minimum of the deviation at $\delta=2 e_{0}$. It is predicted in the CUE case by $(10)$, exact in the limit $\operatorname{Im} e \rightarrow+0$. The zero deviation indicates that the generalized correlator $C\left(e_{0}+\mathrm{i} 0,2 e_{0}\right)$ of any individual spectrum is the same. This is indeed the case; moreover, that spectrum-independent function coincides with the CUE correlator. 


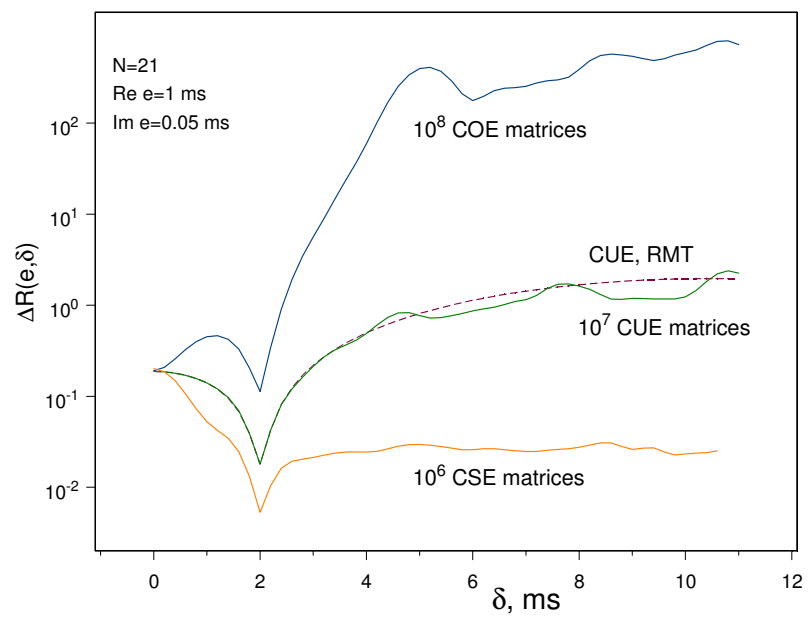

FIG. 9: Deviation of generalized correlator regularized by $\operatorname{Im} e=0.05 \mathrm{~ms}$. Numerical simulation with $10^{8}(\mathrm{COE}), 10^{7}(\mathrm{CUE})$ and $10^{6}$ (CSE) matrices; dashed line depicts RMT prediction for CUE

\section{References}

[1] M. V. Berry. The Bakerian lecture, 1987: Quantum chaology. Proceedings of the Royal Society of London. A. Mathematical and Physical Sciences, 413(1844):183-198, 1987.

[2] M. Sieber and K. Richter. Correlations between periodic orbits and their rôle in spectral statistics. Physica Scripta, 2001(T90):128, 2001.

[3] S. Heusler, S. Müller, A. Altland, P. Braun, and F. Haake. Periodic-orbit theory of level correlations. Phys. Rev. Lett., 98(4):044103, 2007.

[4] S. Müller, S. Heusler, A. Altland, P. Braun, and F. Haake. Periodic-orbit theory of universal level correlations in quantum chaos. New Journal of Physics, 11(10):103025, 2009.

[5] F. Haake. Quantum Signatures of Chaos. Springer Series in Synergetics. Springer, 2010, 2000, 1991.

[6] P. Braun and F. Haake. Chaotic maps and flows: exact Riemann-Siegel lookalike for spectral fluctuations. Journal of Physics A: Mathematical and Theoretical, 45(42):425101, 2012.

[7] A. Pandey. Statistical properties of many-particle spectra: Iii. ergodic behavior in random-matrix ensembles. Annals of Physics, 119(1):170 - 191, 1979.

[8] R. E. Prange. The spectral form factor is not self-averaging. Phys. Rev. Lett., 78:2280-2283, Mar 1997.

[9] U. Smilansky. Semiclassical quantization of maps and spectral correlations. In I. V. Lerner, J. P. Keating, and D. E. Khmelnitskii, editors, Supersymmetry and Trace Formulae: Chaos and Disorder. Proceedings of a NATO ASI held in Cambridge, United Kingdom, September 8-20, 1997, pages 173-192. Plenum Publishers, 1999.

[10] J. B. Conrey, D. W. Farmer, and M. R. Zirnbauer. Howe pairs, supersymmetry, and ratios of random characteristic polynomials for the unitary groups $\mathrm{u}(\mathrm{n})$. pre-print, 2007. arXiv:math-ph/0511024v2.

[11] M. Zirnbauer. Pair correlations of quantum chaotic maps from supersymmetry. In I. V. Lerner, J. P. Keating, and D. E. Khmelnitskii, editors, Supersymmetry and Trace Formulae: Chaos and Disorder. Proceedings of a NATO ASI held in Cambridge, United Kingdom, September 8-20, 1997, pages 153-172. Plenum Publishers, 1999.

[12] M. L. Mehta. Random Matrices. (Pure and Applied Mathematics, Volume 142. Academic Press, 3 Edition, 2004.

[13] K. Życzkowski and M. Kuś. Random unitary matrices. Journal of Physics A: Mathematical and General, 27(12):4235, 1994.

[14] C. Tian and A. I. Larkin. Ehrenfest oscillations in the level statistics of chaotic quantum dots. Phys. Rev. B, 70:035305, Jul 2004.

[15] P. W. Brouwer, S. Rahav, and C. Tian. Spectral form factor near the ehrenfest time. Phys. Rev. E, 74:066208, Dec 2006.

[16] D. Waltner and J. Kuipers. Ehrenfest-time dependence of quantum transport corrections and spectral statistics. Phys. Rev. E, 82:066205, Dec 2010.

[17] K. Życzkowski. Random matrices of circular symplectic ensemble. Marian Smoluchowski Institute of Physics, pre-print, 
1995. http://chaos.if.uj.edu.pl/ karol/pdf/Zy95b.pdf.

[18] This equation can be regarded as the result of replacing $\delta\left(e_{1}-e_{2}\right)$ in the covariance of correlators with real energy arguments 17, 18 by the "smoothed" delta $\eta / \pi\left[\left(e_{1}-e_{2}\right)^{2}+\eta^{2}\right]$, neglecting all other terms and then setting $e_{1}=e_{2}=e$. 